Literature Reviews

\title{
Survey and Comparison of Operating Concept for Routing Protocols in DTN
}

\author{
Kawakib K. Ahmed, Mohd Hasbullah Omar and Suhaidi Hassan \\ Inter Networks Research Lab, School of Computing, \\ University Utara Malaysia, 06010 UUM, Sintok, Kedah, Malaysia
}

Article history

Received: 01-09-2015

Revised: 29-09-2015

Accepted: 26-01-2016

Corresponding Author:

Kawakib K. Ahmed

Inter Networks Research Lab,

School of Computing,

University Utara Malaysia, 06010 UUM, Sintok, Kedah, Malaysia

Email: Kawakib_Khadyair@yahoo.com

\begin{abstract}
Delay Tolerant Network (DTN) are promising techniques to enable data transmission in challenging scenarios where sophisticated infrastructure is not available and the end-to-end path does not exist at the moment of data transmission. These networks are characterized by a long delay, intermittent connectivity and high error rates. Furthermore, the dynamic topology of the network may change randomly. Therefore, routing is one of the most crucial issues that affect the performance of DTN in terms of data delivery, latency and using resources if node mobility is considered. The routing design in DTN raises many challenges to the networks. Therefore, the problem of how to route a packet from one node to another in DTN is of the essence. This paper puts forward a rigorous survey of various routing protocols as well as performs a comparison of diverse routing strategies regarding significant issues in DTN.
\end{abstract}

Keywords: Delay Tolerant Networks, Routing Issues, Routing Techniques

\section{Introduction}

The Transmission Control Protocol/Internet Protocol (TCP/IP) is based on the assumptions, such as symmetrical bi-directional data transfer, continuous endto-end connections, low error rate and low delivery latency. In such case, the TCP/IP has the ability to protect heterogeneous networks. Therefore, with this protocol stack, traditional Internet has achieved a great success. However, there are many types of networks that emerged in recent years. These networks which deployed in the extreme environments are unable to meet the aforementioned assumptions. For example, pocket switched networks, underwater sensor networks, vehicular ad-hoc networks, to mention a few. These special networks are characterized by intermittent connectivity, sparse node density, limited network resources, node mobility and so forth. For these kind of networks, there may never be a complete end-to-end path between the source node and the destination, so traditional TCP/IP protocol is difficult to get efficient achievements. Consequently successful message delivery in such networks faces great challenges as highlighted by (Liu et al., 2012). In this case, Delay Tolerant Network (DTN) concept will provide necessary facility for data transfer. The main difference between Internet and DTN communication is the absence of end-to-end communication path which leads to disconnection, variable delay and high error rate in communication.

DTN uses store-and-forward mechanism to transmit messages from the source node to the destination. A node stores the data in its buffer and then forwards the data to other nodes when a connection is available between two nodes in the network.

The objective of this paper is to highlight the main features of DTN and present an extensive comparison of all routing protocols in these networks. In general, the routing protocols in DTN are categorized according to their characteristics and compare them in terms of mechanism, a number of copies, hop count, advantages and drawbacks.

The paper is organized as follows: In next section the characteristics of DTN are discussed, furthermore, some of DTN applications are explained briefly. DTN architecture, routing issues and routing techniques in DTN are discussed in the subsequent sections.

\section{DTN Characteristics}

DTN is considered as an unstable network topology, long latency, where end-to-end path may not exist and delay may be measured in days for some 
networks. These features make traditional routing protocols for Mobile Ad-hoc Networks (MANET) unacceptable for DTN. This is because most routing protocols for MANET need to set up a continuous path between the source node and destination before any data transmission.

In DTN, it is usually assumed that the link between two nodes does not exist and the latency of the network is not a main concern. The DTN can be widely adopted by challenging networks, for example, mobile sensor networks, military operation networks and space communications. Characteristics of DTN are a perfect match in these challenging networks (Gao et al., 2015). These characteristics include.

\section{Intermittent Connectivity}

DTN is frequently disconnected because of node mobility and energy limitation, which results in a continuous change of network topology. It can be stated that such network holds the case of incomplete and intermittent connectivity so that there is no end-to-end route is guaranteed (Zhang, 2006).

\section{Limited Resources}

DTN nodes have limited resources because of node's mobility. For example, to forward data to the next node the data must be stored in the present node until the connection is established and available. However, more buffer space is required when new data is received or collected. Thus, the limited memory capacity will restrict the data buffering (Mehta and Shah, 2014).

\section{High Delays, Low Data Rate}

The end-to-end delay usually refers to the sum of the total delay of each hop on the route. The delay may be very high because the DTN are intermittently connected; this keeps long time of disconnection and further leading to asymmetric features and a lower data rate (Mehta and Shah, 2014).

The intermittent connectivity, limited resources and high delays, low data rate which hare the main characteristics of DTN, makes routing the main issue in DTN study. The routing in DTN adopts the store-carryand-forward mechanism. In this case, the node stores the messages in its buffer and carry that messages until connection to another node is established.

\section{DTN Applications}

DTN can be widely applied to challenging networks, such as space communications, sparse mobile ad-hoc networks mobile, sensor networks and so on. Characteristics of DTN are a perfect match in these challenging networks.

\section{Inter-Planet Satellite Communication Networks}

The TCP routing protocol was first modified in 1998 to facilitate communications between satellites and emergence of DTN routing protocols. This modification was the goal of the work in collaboration with NASA's Jet Propulsion Laboratory. The main goal of the Interplanetary Networks (IPN) task was to determine the architecture and protocols for interpretation of the Internet at homeland on earth with the other, remotely located on other planets or spacecraft. Basically, architecture of earth's Internet is a network of interrelated networks, so the IPN may be considered of as a network of sporadic Internets. Therefore, the internetworking of such environment requires new techniques to be developed (Karimzadeh, 2011).

\section{Sparse Mobile Ad-Hoc Networks}

These networks may have unexpected intermittent connectivity because of node mobility and sparse deployment. Sometimes sporadic connectivity in these networks can be periodic or predictable. For example, a bus carrying a computer can serve as a store and forward message switch with a limited Radio Frequency (RF) communication capability. As it travels, it provides a form of message switching service to its nearby clients to communicate with distant parties it will visit in the future (Karimzadeh, 2011).

\section{Country-Side Area Networks}

In rural and other environments, DTN can bring digital connectivity with limited or non-existing infrastructure. Cars, buses and boats are considered as transportation systems in these networks, which are utilized to provide the relaying of messages by moving around and collecting/delivering messages from/to various nodes. Recently, a number of projects have exploited such a communication concept. Message ferry project serves as an example; which aims to improve the data delivery system in regions with no existing Internet infrastructure (Zhao et al., 2004). DakNet project is another example that should potentially supply low-cost connectivity to the Internet in villages in India (Karimzadeh, 2011).

\section{Wireless Sensor Networks (WSNs)}

These networks are characterized by resource limitation such as CPU power, memory and energy. Hence, the aim of the communication within these networks is to limit the usage of these resources. The lack of infrastructure may force WSNs gateways to be intermittently connected. There are different reasons that cause the interruption of operable communication links such as interference, environmental hostility, or scheduled downtime (Karimzadeh, 2011). 
DTN routing algorithms can give better performance in many extreme environments than traditional routing protocols, that rely on more stable environments. DTN has recently drawn much attention from researchers due to the wide applications of these networks in challenging environments, such as inter planet satellite communication networks, sparse mobile ad-hoc networks and so on.

\section{DTN Architecture}

The DTN architecture implements store-and-forward message switching by overlaying a new protocol layer called Bundle Layer (Fall, 2003). The Bundle Layer is located between the application layer and the transport layer as shown in Fig. 1. A bundle is known as a message. To increase reliability and to cope with hardware failures, bundles are typically stored in persistent storage.

The Bundle Layer (Scott and Burleigh, 2007) is a store and forward layer, that implements an overlay network providing:

- Custody-based retransmission

- Ability to cope with intermittent connectivity

- $\quad$ Ability to take advantage of scheduled, predicted and opportunistic connectivity (in addition to continuous connectivity)

- Late binding of overlay network endpoint identifiers to constituent internet addresses

The store-and-forward mechanism is like an e-mail system. Along the route from the source node to the destination, the intermediate node holds bundles in storage for a while until the next node becomes available as shown in Fig. 2.

Each node in the network has a storage device such as a hard disk, where the node can store messages. This storage device is called persistent storage as it can store the messages for a long interval of time, unlike short-term memory devices. The importance of the persistent storage appears in cases when the rate of incoming messages is higher than the rate of outgoing messages, or when the next node is not available for a very long time (Minz, 2012). Each node in DTN might be a router, host, or gateway. These entities act as a source, forwarder, or destination (Fall, 2003).

\section{Router}

A router forwards each bundle to another node in the same DTN region and may optionally support custody transfer. The router requires storage to store incoming packets before forwarding these to outgoing links because:

- There is no guarantee that next hop link is currently available

- Asymmetric data rate between sender and receiver

- Retransmission due to the high error rate link. When a message is transmitted toward the destination, it may need to retransmit again in case an error occurred at the upstream node, or when the upstream node declines acceptance of a forwarded message (Warthman, 2012)

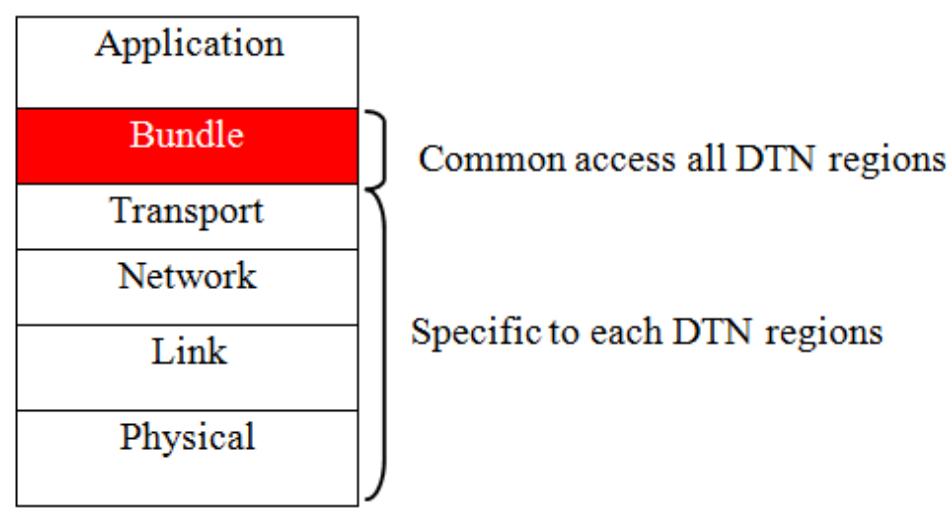

Fig. 1. DTN architecture (adopted from Arora and Singh (2014))

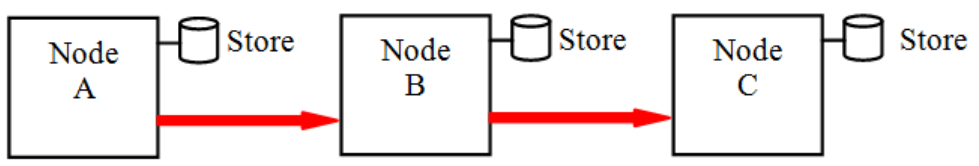

Fig. 2. Store-and-forward mechanism in DTN architecture (adopted from Warthman (2012)) 


\section{Host}

A host sends or receives bundles (i.e., it is the source and/or destination of the bundle transfer) and requires storage to queue bundles. It needs an optional custody transfer capacity for retransmission (Warthman, 2012).

\section{Gateway}

A gateway considers as interconnection point that forwards bundle to other DTN regions with different protocols by supporting interoperability. To perform mapping between different transports layers, the gateway should have storage for reliable delivery. The gateway also checks arriving data before forwarding it and performs authentication (Warthman, 2012).

\section{Routing Criteria in DTN}

Routing protocols for Mobile Ad-hoc Networks (MANET) are built with the assumption that the network is fully connected; i.e., the path always exists between each node in the network, or the path that fails for a very short period of time. Therefore, routing protocols utilized in MANET are not convenient to work in DTN. As a result, before sending any data; it is necessary to find a complete route between the sender node and the receiver node. These protocols will not succeed in transmitting any data if the route between the source node and the receiver is not available (Herbertsson, 2010) as shown in Fig. 3.

Routing protocols in DTN can be differentiated by queue management in terms of the amount of information available when making the forwarding decisions and the number of destination a message can have. DTN routing protocols exploit node mobility and message buffering to cope with problems in the network such as intermittent connection and partitions. This makes it possible for a node to carry messages and thus bridge partition in the network (Herbertsson, 2010). The properties of DTN certainly raise a number of interesting issues in routing (Shen et al., 2008). These properties include:

\section{Routing Objective}

Maximizing message delivery and minimizing resource consumption (i.e., energy, buffer space and network bandwidth) are the most important objectives in DTN.

\section{Resource Allocation}

The routing protocols for DTN should maintain the stability between the goals of minimizing resource consumption and maximizing message delivery, which may conflict with each other. For example, it is unnecessary to store copies of the data to all hosts on the network, unless we can ensure that data can be delivered to the destination. On the other hand, it maximizes the probability that the specified message is finally delivered by increasing the number of message copies at multiple hosts.

\section{Buffer Space}

In order to deal with the lengthy disconnection time, messages must be stored in a buffer for long intervals. Intermediate routes need additional buffer space to save all the messages that should be transmitted. Besides that, there is a relationship between the number of pending messages (not delivered yet to the destination) and buffer space. Consequently, a higher number of pending messages means higher buffer space.

\section{Reliability}

To guarantee stable and successful delivery of data, routing protocols in DTN should have some knowledge of reliable data delivery. For example, messages should have some acknowledgment carried back from the receiver node to the source node, when a message finally arrives at the receiver node for later use.

\section{Energy}

Nodes in DTN usually suffer from low energy due to node mobility and the difficulties of connection to the power station. Much of the energy is used for message routing. The consumed energy is utilized in the sending, receiving of messages and performing computations as well as message storing. Therefore, it is necessary to design energy efficient routing protocols.

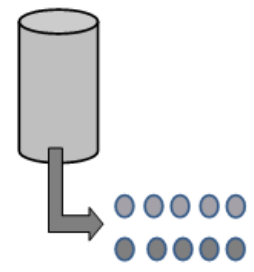

Common Links between routers are not always reliable and available

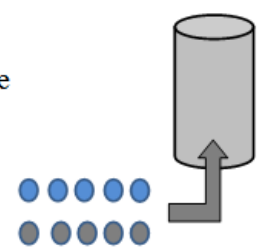

Fig. 3. Routing problem in DTN (adopted from Nikunj (2014)) 
The concept of DTN emerged when the traditional routing protocols failed to work in the extreme environment. The extreme environment characterised by frequent interruption, no constant end-to-end connectivity and limited resources. Therefore, routing of the messages in DTN is mainly based on the storeand-forward mechanism. That is, when a node receives a message and there is no continuous end-toend path to the destination node, the message is buffered in the current node till it encounters other nodes. Thus, routing in DTN is one of the major issues affecting the overall performance of DTN networks in terms of data delivery and resource consumption.

\section{Routing Techniques in DTN}

Routing in DTN is a big challenge because of frequency and length of the disconnection time between nodes in the network (Ali et al., 2010). However, the main role of routing in DTN is to find an opportunity to connect nodes and to transmit data between them when the nodes meet each other if possible. Furthermore, an efficient routing protocol should be simple, scalable and capable of working at both low and high message load. Moreover, it should have optimal delivery probability, low delay and low overhead ratio (Supriya and Pramila, 2014). There are three routing techniques in DTN. These include opportunity routing techniques, prediction routing techniques and message ferry routing techniques as illustrated in Fig. 4.

\section{Opportunity Routing Techniques}

In this approach, messages are forwarded randomly hop by hop with the assumption of final delivery of messages. In this technique, nodes exchange messages only at the same place when the nodes meet. In order to increase the opportunity of message delivery, multiple copies of the same messages are flooded in the network (Cabacas et al., 2014) such as Epidemic (Vahdat and Becker, 2000) and Spray and Wait (Spyropoulos et al., 2005).

\section{Epidemic Routing Protocol}

The Epidemic Routing (ER) was proposed by Vahdat and Becker (2000) aims to deliver messages in mobile ad-hoc networks where there is no guarantee of the continuous path between the source node and destination. The ER was suggested for the random exchange of pair-wise messages among mobile hosts to ensure final messages delivery. This protocol has many advantages such as: Decrease in message latency and increase in delivery rate.

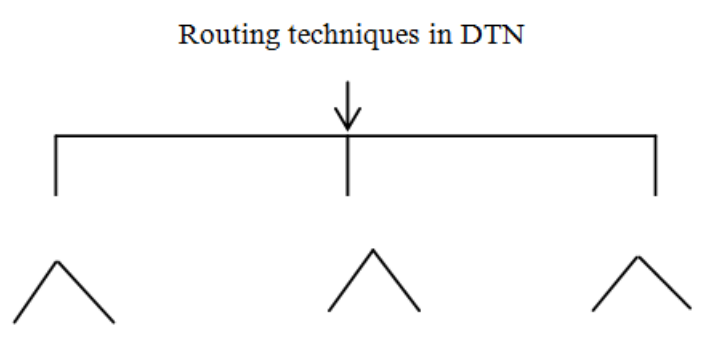

Fig. 4. Routing techniques in DTN

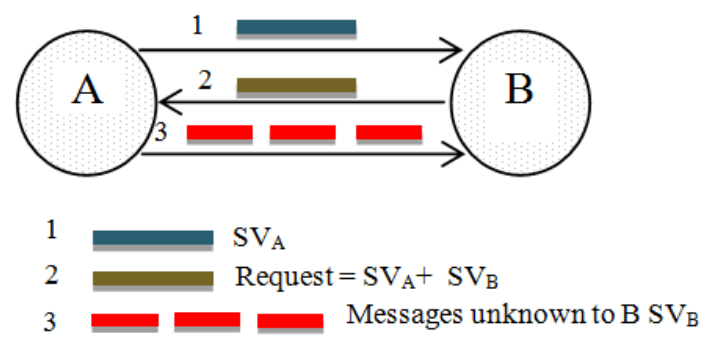

Fig. 5. Message exchange in epidemic (adopted from Vahdat and Becker (2000))

To deliver messages to their destination, ER provides a redundant number of random messages exchange. This leads to guaranteeing the destination node receiving the messages in anyway. During a contact between nodes in ER, every node stores all messages that have been transmitted. Each node in the network holds a list of all messages in the database called Summary Vector (SV). The SV is first exchanged when two nodes meet and only messages that does not existed in the other $\mathrm{SV}$ are exchanged as in Fig. 5. In the case of small message size and in very sparsely networks, the ER strategy is almost possible.

The most crucial problem of the ER approach is that even when the messages are successfully delivered to their destination, it continues to spread in the network. The limitation of this technique is that it has to perform a large amount of redundant work, since all nodes in the network will receive each and every message. This will undesirably affect buffer utilization. However, it enhances the probability of message transfer during network failure. In addition to this, it minimizes the amount of time required to deliver the message successfully (Spyropoulos et al., 2005).

To optimize ER performance, a number of studies have been carried out in this field. Ayub et al. (2010) proposed a new message forwarding technique by studying the impact of packet forwarding order with ER to optimize its performance in terms of delivery probability. In the same field and to enhance the performance of ER, Rashid et al. (2011) study the impact of buffer management Drop Largest (DLA). The study described how to drop large-size message when the node buffer is congested with five mobility models. 
On the other hand, Abdulla and Simon (2008) presented multicasting in DTN using controlled flooding schemes and proposed Controlled Epidemic Routing for Multicasting (CERM) in DTN. The basic mechanisms used in CERM are message expiration times, TTLs, forwarding probabilities and the number of copies to spread. Ramanathan et al. (2007) presents a new protocol for routing in DTN called Prioritized Epidemic Routing Protocol (PREP). The proposed PREP uses expiry time information and topology awareness to decide which bundles hold back data and which bundles to delete in case of resource consuming.

Since ER is known to consume lot of network resources (buffer space and bandwidth), this protocol is appropriate for animal monitoring networks such as Zebra Net and Shared Wireless Infostation Model (SWIM), where random mobility patterns and contacts cannot be predicted (Spyropoulos et al., 2004; Suganthe and Balasubramanie, 2008). As the number of duplicated messages will be very large, this routing protocol is not useful for dense networks. The main shortcoming of this protocol is as follows: Wasting resources (power, buffer and bandwidth) and in case of limited resources, it causes contentions, as well as leading messages to drop. However, some studies such as Vahdat and Becker (2000) show that ER is capable of delivering all transmitted messages. Studies that have assumed unlimited or sufficiently large buffer sizes at each node are imaginative assumptions. If the amount of exchanged messages exceeds the actual buffer space limit, it will cause the entire network to stop in the case of random routing. To avoid this waste of buffer size, a form of controlled flooding protocol known as Spray and Wait has been proposed.

\section{Spray and Wait Routing Protocol}

Spray and Wait (SnW) is a routing protocol which was developed by researchers at the University of Southern California to control the number of redundant messages in DTN (Haris, 2010). This protocol follows the same strategy as ER, by forwarding random copies of the message to other nodes in the mobile network during contact. However, there is a difference between ER and $\mathrm{SnW}$ where SnW protocol restricts the complete number of spread copies of the same message to a constant number of L (specific relays).

The SnW approach consists of two phases (spray phase and wait phase). In the spray phase, L copies of the message are created by the source node. Messages are thus transmitted by the source node itself; other nodes receive the message until the total number of $\mathrm{L}$ is reached. In the wait phase, all L nodes store a copy of the message to achieve direct transmission (Shah et al., 2003). To facilitate performances of the algorithm Spyropoulos et al. (2005) proposed the Binary Spray and Wait (BSW) scheme. This method provides the best results if all the nodes' mobility patterns in the network are Independent and Identically Distributed (IID) with the same probability distribution (Mehta and Shah, 2014).

On the other hand, Sammou (2012a) proposed a new routing protocol called spray and dynamic to improve the performance of DTN. This also considered as improvement of spray and wait routing protocol by combining two protocols: MaxProp and the model of "transfer by delegation" (Custody Transfer).

Patel et al. (2013) has proposed an opportunistic routing protocol with enclosed message copies, called the Vibrant Energy-aware Spray and Wait (VESW), that utilizes the information about vibrancy of node and remaining energy to allocate the number of copies between the corresponding pair nodes in the spray phase.

To avoid random forwarding in SnW, Liu et al. (2012) proposed an algorithm named Relay-probabilitybased Adaptive Spay and Wait (R-ASW). The R-ASW uses the performance of receiver nodes to determine whether forward message to the encountered node and calculate a number of message copies to be forwarded. The proposed algorithm has better performances and it is suitable for DTN as the results shown.

Al Hinai et al. (2012) proposed a new protocol, called Trust-Based Spray and-Wait (TB-SnW), based on trust management. The basic idea of the protocol is to let each node maintain a trust list for all other nodes it meets and use the trust level to mitigate attacks. In order to distinguish the true ones from black holes, TB-SnW takes advantage of the previous behavior of nodes and provides a dynamic mechanism to assign replicas to nodes.

Table 1. Comparison between opportunistic techniques

\begin{tabular}{lll}
\hline Protocol & Epidemic & Spray and Wait \\
\hline Mechanism & Flooding. & Flooding. \\
No. of Copies & Unlimited. & N-Copies. \\
Metrics & 1-Average Latency. & 1-Delivery probability. \\
& 2-Bandwidth. & 2-Latency overhead. \\
Hop Count & 3-Buffer consumption. & 3-Buffer time average. \\
Drawbacks & One & Multiple. \\
& High resources consumption. & 1-Random decision making. \\
& & 2- Relay nodes wait until it encounters the destination. \\
Advantages & 1-Find the optimal path. & 3-Nodes must keep track of other nodes movement. \\
& 2-Small delay. & Control level of flooding. \\
\hline
\end{tabular}


The opportunity techniques have different advantages and drawbacks as outlined in Table 1. ER, which is an uncontrolled forwarding based technique, has the smallest delay at a very high cost of network resources and a higher delivery ratio (Vahdat and Becker, 2000). The spray phase of SnW decrease buffer space consumption and ER bandwidth by limiting the number of forwarded messages. Furthermore, using the transitively calculated utility function; the spray and focus routing protocol enhances the selection of the message forwarders (Spyropoulos et al., 2007).

\section{Prediction Techniques}

These techniques include the Probabilistic Routing Protocol using History of Encounters and Transitivity (PRoPHET) (Lindgren et al., 2003) and MaxProp (Burgess et al., 2006).

\section{PRoPHET Routing Protocol}

Lindgren et al. (2003) have developed PRoPHET which it works as similar as the ER protocol. This routing protocol attempts to decrease the use of resources and tries to keep the best routing capabilities of ER by forwarding messages to selected nodes only, rather than forwarding messages to all nodes in the network. Ametric called Delivery Predictability (DP) was introduced by Lindgren et al. (2004), where, $P(A$, $B) \in[0,1]$ which is computed at each node A for every known destination $B$. The node with higher DP value for a specific destination is supposed to be a better path for message delivery to that destination (i.e., if $P(A, B)>$ $P(C, D)$ ); message for receiver node $\mathrm{B}$ is preferred to forward to node $\mathrm{A}$ rather than the node $\mathrm{C}$. The calculation of the $P(A, B)$ may be different from $P(B, A)$ because routes in DTN are not symmetry. The DP is always updated for each node when two nodes are comunicating. If the node $\mathrm{B}$ has not communicated with node $\mathrm{A}$ for a long time or has never connected to node $\mathrm{B}$, such that $P(A, B)<P_{-}$threshold then $P_{-}(A, B)$ should be set to $P_{-}$init. $P$ init should be set to 0.5 (Lindgren et al., 2004). The DP is calculated in three steps (Lindgren et al., 2003; Sammou, 2012b):

When a node A meets another node B: A updates the probability of delivery as shown in the following Equation 1:

$$
P(A, B)=P(A, B)_{\text {old }}+\left(1-\partial-P(A, B)_{\text {old }} * P_{\text {init }}\right)
$$

where, $P_{\text {init }} \in[0,1]$ is an initialization constant.

For nodes infrequently met by A: A updates the probability of delivery in accordance with the following Equation 2:

$$
P(A, B)=P(A, B)_{\text {old }} * \gamma^{k}
$$

where, $\gamma \in[0,1]$ is the aging constant and $\mathrm{k}$ is the number of time units that have elapsed since the last time the metric was aged.

For a node $\mathrm{C}$ known by a node $\mathrm{B}$ : A updates the probability of delivery in accordance with the following Equation 3:

$$
P(A, C)=\operatorname{Max}\left(P(A, C)_{\text {old }}, P(A, B)^{*} P(B, C)^{*} \beta\right)
$$

where, $\beta \in[0,1]$ is a scaling constant that decides how large impact the transitivity should have on the delivery predictability.

Sok and Kim (2013) have proposed routing protocol to enhance the performance of the PRoPHET protocol. This protocol used a distance metric as an additional factor to produce best DP. On the other hand, Borah (2012) has extended the PRoPHET routing protocol by using fuzzy logic. In this approach, the DP is obtained by using two metrics; energy value and delivery predictability in order to mitigate the delay in message delivery and to minimize resource consumption in the network.

On the other hand, Mehto and Chawla (2014) proposed Different Neighbor History-Spray and Wait (DNH-SaW) aims to improve the wait phase based on DP using PRoPHET. This protocol calculates the number of message copies to be forwarded based on the performance of the receiver node in spray phase and in the wait phase the waiting node uses PRoPHET for transmission.

The problem of PRoPHET is the relationship between the overhead ratio and number of nodes; as the number of nodes increases the overhead ratio increases. This protocol is known for its complexity of the forwarding strategy. Thus, it consumes a lot of resources to process and store historical values. If a node receives a message and there is no path to the destination, node buffers that message and forwards it whenever another node is discovered. At this point, the forwarding decision could be affected by numerous issues. For example, forwarding more copies of the received messages result in higher delivery probability values, lower delivery delays and more resources spent. PRoPHET is the only DTN routing protocol that has been formally documented using RFC drafts (Lindgren et al., 2009).

\section{MaxProp}

MaxProp (Burgess et al., 2006) is a flooding-based routing protocol. This protocol uses several mechanisms to reduce delivery latency and to improve the delivery rate. The MaxProp routing protocol uses local information and node mobility to select the best hope for messages delivery. The buffer of MaxProp is divided into two phases. Firstly, messages are stored from low to high based on hop count information. 


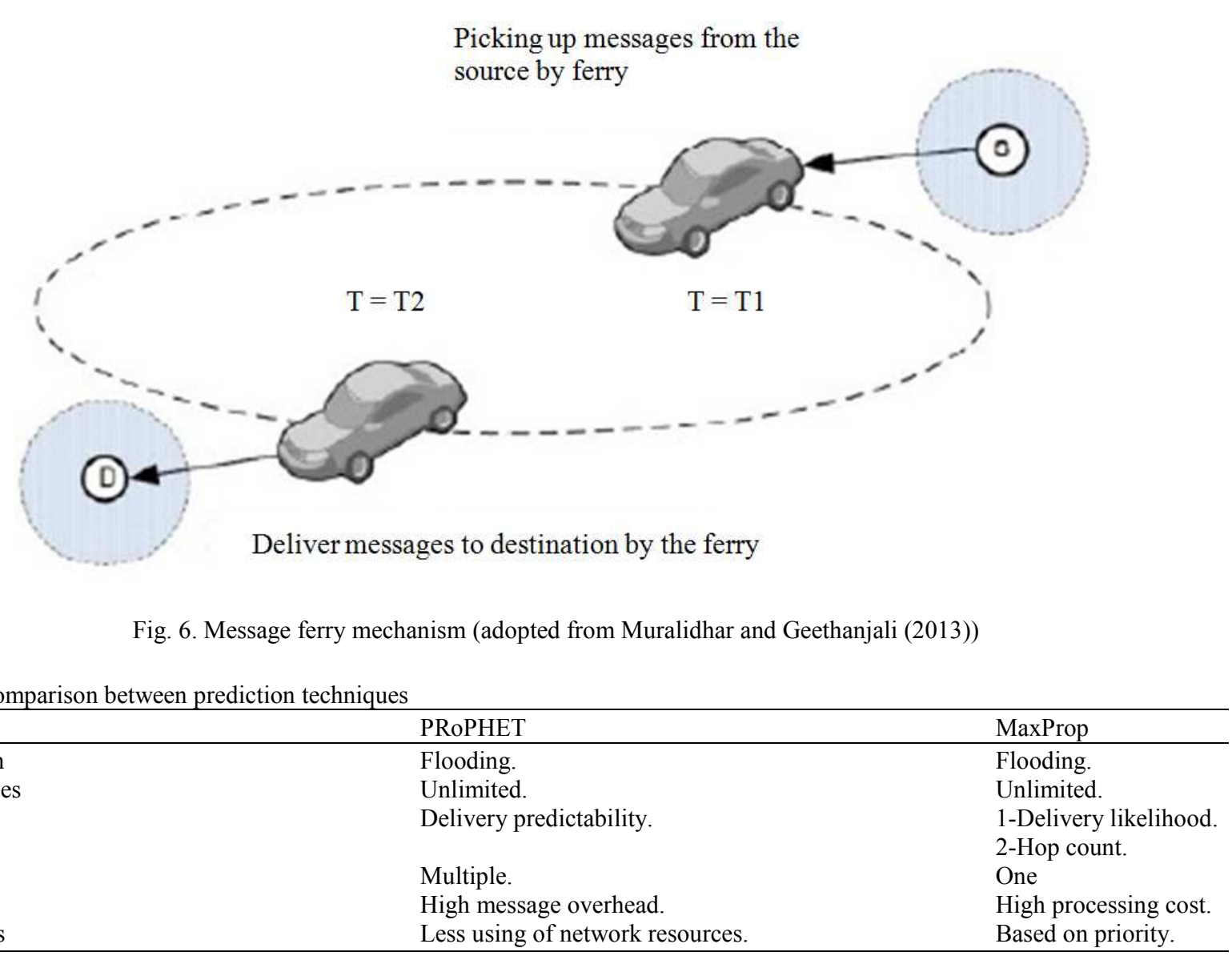

Table 2. Comparison between prediction techniques

\begin{tabular}{lll}
\hline Protocol & PRoPHET & MaxProp \\
\hline Mechanism & Flooding. & Flooding. \\
No. ofCopies & Unlimited. & Unlimited. \\
Metrics & Delivery predictability. & 1-Delivery likelihood. \\
& & 2-Hop count. \\
Hop Count & Multiple. & One \\
Drawbacks & High message overhead. & High processing cost. \\
Advantages & Less using of network resources. & Based on priority. \\
\hline
\end{tabular}

Secondly, messages are ordered by cost from high to low. The buffer is used from both ends. The first phase uses the front end of the buffer while the second phase uses the back end of the buffer. The MaxProp routing protocol is designed for vehicle-based delay tolerant networks. This protocol forwards messages to any node in the network that has the highest probability to deliver the messages to their final destination (Burgess et al., 2006). The MaxProp protocol has low performance when nodes have small buffer sizes because of the adaptive threshold calculation, but it gives better performance with larger buffer size.

The prediction techniques as outlined in Table 2 , attempt to mitigate buffer contention and message overhead by forwarding messages to nodes with high delivery predictability only. It may take a very long time before each node receives the delivery predictability of other nodes because of disconnect nature of the networks. Moreover, in case of big networks and as mentioned in (Spyropoulos et al., 2004), the source node may take longer time before finding a message forwarder with high delivery predictability to the destination. Which called the slow start problem.

\section{Message Ferry Techniques}

In these techniques, additional mobile nodes are used. These nodes are known as ferries and used for message delivery in the network. The route of these ferries are controlled to increase the delivery performance by using the store-and-carry mechanism (Cabacas et al., 2014). These techniques include the Message Ferry (MF) routing protocol (Zhao et al., 2004) and Meet and Visit (MV) (Burns et al., 2005).

\section{Message Ferry Routing Protocol}

Message Ferrying (MF) approach was first described by Zhao et al. (2004). These message ferries allow nodes to communicate when the network is disconnected and when nodes do not have global knowledge of the network. It is a proactive routing algorithm created to address network partitions in intermittently connected ad hoc networks by establishing non-random in node movement as shown in Fig. 6. The MF approach can be utilized in various applications such as wide area sensing, non-interactive, battlefield, anonymous and disaster relief. In some scenarios such as earthquakes, ground vehicles or unmanned aerial vehicles which are equipped with short-range radios and large-storage can 
be used as MF to carry and gather data between disconnected areas. This enables victims and rescue participants to use devices such as smart tags, PDAs, cell phones for communication (Zhao et al., 2004).

Depending on whether ferries or nodes initiate non-random proactive movement, Zhao et al. (2004) developed two variations of MF. These two variations include Node-Initiated MF (NIMF) scheme and FerryInitiated MF (FIMF) scheme. In order to reduce delay and packet loss ratio, Xue et al. (2012) has proposed an algorithm named Adaptive Message Ferry (ADMF) routing algorithm. The ADMF algorithm has the ability to dynamically choose a single-ferry and multiple-ferry mechanism according to variable network traffic. When the traffic is higher, the ADMF algorithm can improve network throughput based on the simulation results.

On other hands, a routing scheme using the MF technique was extended for disconnected vehicular ad-hoc networks by $\mathrm{Yu}$ and Ko (2009). In this technique, to ensure 1-hop communication between vehicles, geographic information was used to control the block size and to divide the road into blocks. Speed selection was designed for fast packet delivery and minimum number of ferries. The authors in (Chuah and Yang, 2006) designed a Node Density Based Adaptive Routing (NDBAR) scheme that allows regular nodes to volunteer to be message ferries when there are very few nodes around them to ensure the feasibility of continued communications. The NDBAR scheme can achieve the highest delivery ratio in very sparse networks that are prone to frequent disruptions.

\section{Meet and Visit (MV)}

Burns et al. (2005) proposed the MV algorithm, which is based on observed meetings between peers and visits of peers to geographic locations. The name MV protocol itself comes from Meetings and Visits. As in ER, messages are exchanged during the contact between nodes. However, this protocol is a more flexible method to choose the messages to be forwarded to an encountered node.

Basically, to deliver messages to their final destinations successfully, the choice was depending on the probability of encountered nodes. The delivery probability is based on recent past notifications of both the meetings between nodes and the visits of nodes to geographical locations.

This scheme gives information about a meeting of the intermediate node and location. Knowledge of meetings and visit places is stored in every intermediate node and is used to estimate message delivery probability. A similar approach is followed in the PRoPHET routing protocol (Lindgren et al., 2003). Three assumptions are available in the MV protocol that includes (Karimzadeh, 2011):

- Destination nodes are fixed

- Infinite link capacity

- Unlimited buffer space

These techniques might be effective in terms of buffer consumption and message overhead, it is necessary for ferries to change their paths on demand to help other nodes to deliver messages. In addition, message ferry techniques are complicated and costly in terms of resources that are not linked to communication as compared to other techniques. However, the overall performance of the system might be improved in terms of delays and metrics in a drastic manner. A comparison between message ferry techniques outlined in Table 3.

\section{Research Trends}

Current Internet engulfing activities has shown the high interconnection of devices, smart gadgets and other wireless communication platforms for information dissemination over the Internet. This has created a need for DTN. As one of the leading research open issue is routing in DTN (Zhang et al., 2014). Routing therefore provides the platform for data and message traversing on the Internet due to the delays, queues and disconnectivity. It is thus safe to say that currently there has not been a full polynomial solution to routing operation in DTN. Current studies have shown that routing in DTN uses a bundle protocol specification for information dissemination. According to Scot and Burleigh (2007), a full description of the bundle was presented in RFC 5050.

Table 3. Comparison between message ferry techniques

\begin{tabular}{lll}
\hline Protocol & MF & MV \\
\hline Mechanism & Forwarding. & Forwarding. \\
No. of Copies & Single. & Multiple. \\
Metrics & 1-Delivery rate. & Ferry route. \\
& 2-Energy consumption. & Long message delay. \\
\hline
\end{tabular}


The DTN nature of unpredictive link fluctuation makes the routing protocol, one of the leading open issues. Thus, in order to mitigate the loss of data and information, there is a need to propose DTN routing concept to fits the problem appropriately. Studies have been able to categorise the methods as opportunistic, predictiction and the ferry routing protocols. For better routing in DTN, several dependant factors need to be taken into consideration. Amongst the factors that are dependant on information is the node history also referred to as "future contacts".

Sometimes in DTN it is possible to predict when coming contact can be, like the planetary motion. The nature of motion of planets is such that using laws of motion and physics, a highly accurate estimate can be made regarding the next contact.

However, in some unique cases, it is not possible to predict when the next contacts between nodes will happen such as disaster relief operations Nelson et al. (2007).

Another factor that affects the contact predictory estimation is mobility. Mobility has been seen as a leading issue that needs adequate concept, approach and some time good algorithm to palliate its effects. With the wide range in usage of mobile devices, mobility is thus an unavoidable variable. The movement of node in and out of network in exponential intervals makes contact is some time difficult. In a static network where nodes are on a finite number, it is easier to establish the contact depending on the quality of the channels. This makes the transfering of data from one location to another easily achievable. Jea et al. (2005) suggested that high availability of the mobile nodes guarantee better chances of successful data delivery and sharing.

Consequently, in vehicular network, the nodes are actively mobile thus giving more options for routing in deciding the path to traverse on (Balasubramanian et al., 2007; Spyropoulos et al., 2005; 2007).

The third significant factor is the availability of network resources. It is possible that many nodes, like mobile devices and mobile phones, are limited in terms of storage space, transmission rate and battery life. Others, such as buses on the road, may not be as limited. Therefore routing protocols would utilize this information to optimally determine how messages should be transmitted and stored so as to reduce the burden of limited resources (Haris, 2010).

\section{Conclusion}

The major challenge in DTN is how to enable efficient communication for the intermittently connected environment or partition based network where the nodes are sparsely distributed. The aim of this paper is to detail the general information needed about DTN characteristics and architecture. The paper also discussed in more details some of the routing issues and classifications of routing protocols. Each routing protocols classification has its own advantages and drawbacks that were deliberated in the comparative table. Therefore, this paper presented opens issues in DTN routing as a guide to vending into DTN routing research to achieve standardization.

\section{Funding Information}

The authors wish to thank the Ministry of Education, Malaysia for funding this study under the Long Term Research Grant Scheme (LRGS/bu/2012/UUM/Teknologi Komunikasi dan Infomasi).

\section{Author's Contribution}

All the authors equally contributed in this work and the article.

\section{Ethics}

This article is original and contains unpublished material. The corresponding author confirms that all of the other authors have read and approved the manuscript and no ethical issues involved.

\section{References}

Abdulla, M. and R. Simon, 2008. Controlled epidemic routing for multicasting in delay tolerant networks. Proceedings of the IEEE International Symposium on Modeling, Analysis and Simulation of Computers and Telecommunication Systems, Sept. 08-10, IEEE Xplore Press, Baltimore, MD., pp: 1-10. DOI: 10.1109/MASCOT.2008.4770551

Al Hinai, A., H. Zhang and Y. Chen, 2012. Mitigating blackhole attacks in delay tolerant networks. Proceedings of the 13th International Conference on Parallel and Distributed Computing, Applications and Technologies, Dec. 14-16, IEEE Xplore Press, Beijing, pp: 329-334.

DOI: 10.1109/PDCAT.2012.95

Ali, S., J. Qadir and A. Baig, 2010. Routing protocols in delay tolerant networks-a survey. Proceedings of the 6th International Conference on Emerging Technologies, Oct. 18-19, IEEE Xplore Press, Islamabad, pp: 70-75.

DOI: $10.1109 /$ ICET.2010.5638377

Arora, H. and G. Singh, 2014. Survey on probabilistic routing schemes in Delay Tolerant Networks (DTNs). Int. J. Applic. Innovat. Eng. Manage., 3: 81-85.

Ayub, Q., S. Rashid and M.S.B.M. Zahid, 2010. Optimization of epidemic router by new forwarding queue mode TSMF. Int. J. Comput. Applic., 7: 5-8. DOI: $10.5120 / 1294-1781$ 
Balasubramanian, A., B. Levine and A. Venkataramani, 2007. DTN routing as a resource allocation problem. ACM SIGCOMM Comput. Commun. Rev., 37: 373-384. DOI: $10.1145 / 1282427.1282422$

Borah, J., 2012. Application of computational intelligence paradigm in the probabilistic routing for intermittently connected network. IOSR J. Comput. Eng., 8: 32-36. DOI: 10.9790/0661-0823236

Burgess, J., B. Gallagher, D. Jensen and B.N. Levine, 2006. MaxProp: Routing for vehicle-based disruption-tolerant networks. Proceedings of the 25th IEEE International Conference on Computer Communications, Apr. 23-29, IEEE Xplore Press, Barcelona, Spain, pp: 1-11. DOI: 10.1109/INFOCOM.2006.228

Burns, B., O. Brock and B.N. Levine, 2005. MV routing and capacity building in disruption tolerant networks. Proceedings of the IEEE 24th Annual Joint Conference of the IEEE Computer and Communications Societies, Mar. 13-17, IEEE Xplore Press, pp: 398-408. DOI: 10.1109/INFCOM.2005.1497909

Cabacas, R.A., H. Nakamura and I.H. Ra, 2014. Energy consumption analysis of delay tolerant network routing protocols. Int. J. Software Eng. Applic., 8: 1-10.

Chuah, M. and P. Yang, 2006. Node density-based adaptive routing scheme for disruption tolerant networks. Proceedings of the IEEE Military Communications Conference, Oct. 23-25, IEEE Xplore Presss, Washington, DC., pp: 1-6. DOI: 10.1109/MILCOM.2006.302040

Fall, K., 2003. A delay-tolerant network architecture for challenged internets. Proceedings of the Conference on Applications, Technologies, Architectures and Protocols for Computer Communications, Aug. 25-29, ACM, Karlsruhe, Germany, pp: 27-34. DOI: $10.1145 / 863955.863960$

Gao, L., S. Yu, T.H. Luan and W. Zhou, 2015. Delay Tolerant Networks. 1st Edn., Springer, Cham, ISBN-10: 3319181084, pp: 85.

Haris, A., 2010. A DTN study: Analysis of implementations and tools. MSc Thesis, Technical University of Denmark.

Herbertsson, F., 2010. Implementation of a delaytolerant routing protocol in the network simulator NS-3. Department of Computer and Information Science.

Jea, D., A. Somasundara and M. Srivastava, 2005. Multiple controlled mobile elements (data mules) for data collection in sensor networks. Proceedings of the 1st IEEE International Conference on Distributed Computing in Sensor Systems, Jun. 30-Jul. 1, Springer, Marina del Rey, CA, USA, pp: 244-257. DOI: 10.1007/11502593_20
Karimzadeh, M., 2011. Efficient routing protocol in Delay Tolerant Networks (DTNs).

Lindgren, A., A. Doria and O. Schelén, 2003. Probabilistic routing in intermittently connected networks. ACM SIGMOBILE Mobile Comput. Commun. Rev., 7: 19-20. DOI: $10.1145 / 961268.961272$

Lindgren, A., A. Doria and O. Schelén, 2004. Probabilistic routing in intermittently connected networks. Proceedings of the $1 \mathrm{st}$ International Workshop on Service Assurance with Partial and Intermittent Resources, Aug. 1-6, Springer, Fortaleza, Brazil, pp: 239-54. DOI: $10.1007 / 978-3-540-27767-5$ _24

Lindgren, A., A. Doria, E. Davies and S. Grasic, 2009. Probabilistic routing protocol for intermittently connected networks draft-irtf-dtnrg-prophet-09. Research Task Force.

Liu, J., M. Tang and G. Yu, 2012. Adaptive spray and wait routing based on relay-probability of node in DTN. Proceedings of the International Conference on Computer Science and Service System, Aug. 11-13, IEEE Xplore Press, Nanjing, pp: 1138-1141.

DOI: $10.1109 /$ CSSS.2012.288

Mehta, N. and M. Shah, 2014. Performance of efficient routing protocol in delay tolerant network: A comparative survey. Int. J. Future Generat. Commun. Network., 7: 151-158. DOI: $10.14257 /$ ijfgen.2014.7.1.15

Mehto, A. and M. Chawla, 2014. Modified different neighbor history spray and wait using PROPHET in delay tolerant network. Int. J. Comput. Applic., 86: 30-35. DOI: $10.5120 / 15088-3457$

Minz, B., 2012. Routing in delay tolerant networks. Bachelor of Technology, National Institute of Technology Rourkela, India.

Muralidhar, K. and N. Geethanjali, 2013. A novel ferryinitiated message ferrying approach for data delivery in disconnected mobile ad hoc networks through native ferries. Int. J. Eng. Sci. Res., 4: 1022-1027.

Nelson, S.C., A.F. Harris and R. Kravets, 2007. Eventdriven, role-based mobility in disaster recovery networks. Proceedings of the 2nd ACM Workshop on Challenged Networks, Sept. 9-14, ACM, Montreal, Canada, pp: 27-34. DOI: $10.1145 / 1287791.1287798$

Nikunj, D., 2014. Comparative study of routing protocols in delay tolerant networks. Int. J. Adv. Res. Electr. Electron. Instrument. Eng., 3: 7242-7246.

Patel, V.G., T.K. Oza and D.M. Gohil, 2013. Vibrant energy aware spray and wait routing in delay tolerant network. J. Telemat. Informat., 1: 43-47. DOI: $10.12928 /$ jti.v1i1.43-47 
Ramanathan, R., R. Hansen, P. Basu, R. Rosales-Hain and R. Krishnan, 2007. Prioritized epidemic routing for opportunistic networks. Proceedings of the 1st International MobiSys Workshop on Mobile Opportunistic Networking, Jun. 11-14, ACM, USA., pp: 62-66. DOI: 10.1145/1247694.1247707

Rashid, S., Q. Ayub, M.S.M. Zahid and A.H. Abdullah, 2011. Impact of mobility models on DLA (drop largest) optimized DTN epidemic routing protocol. Int. J. Comput. Applic., 18: 35-39.

Sammou, E.M., 2012a. Efficient probabilistic routing in delay tolerant networks. Proceedings of the International Conference on Multimedia Computing and Systems, May 10-12, IEEE Xplore Press, Tangier, pp: 584-589.

DOI: 10.1109/ICMCS.2012.6320290

Sammou, E.M., 2012b. Spray and dynamic: Advanced routing in delay tolerant networks. Int. J. Commun. Netw. Syst. Sci., 5: 98-104. DOI: $10.4236 /$ ijens.2012.52013

Scott, K.L. and S. Burleigh, 2007. Bundle protocol specification.

Shah, R.C., S. Roy, S. Jain and W. Brunette, 2003. Data mules: Modeling and analysis of a three-tier architecture for sparse sensor networks. Ad Hoc Netw., 1: 215-233. DOI: $10.1016 / \mathrm{S} 1570-8705(03) 00003-9$

Shen, J., S. Moh and I. Chung, 2008. Routing protocols in delay tolerant networks: A comparative survey. Proceedings of the 23rd International Technical Conference on Circuits/Systems, Computers and Communications, (SCC' 08), pp: 6-9.

Sok, P. and K. Kim, 2013. Distance-based PRoPHET routing protocol in disruption tolerant network. Proceedings of the International Conference on ICT Convergence, Oct. 14-16, IEEE Xplore Press, Jeju, pp: 159-164. DOI: 10.1109/ICTC.2013.6675329

Spyropoulos, T., K. Psounis and C.S. Raghavendra, 2004. Single-copy routing in intermittently connected mobile networks. Proceedings of the 1st Annual IEEE Communications Society Conference on Sensor and Ad Hoc Communications and Networks, Oct. 4-7, IEEE Xplore Press, pp: 235-244. DOI: $10.1109 /$ SAHCN.2004.1381922

Spyropoulos, T., K. Psounis and C.S. Raghavendra, 2005. Spray and wait: An efficient routing scheme for intermittently connected mobile networks. Proceedings of the ACM SIGCOMM Workshop on Delay-Tolerant Networking, Aug. 22-26, ACM, Philadelphia, PA, USA., pp: 252-259.

DOI: $10.1145 / 1080139.1080143$
Spyropoulos, T., K. Psounis and C.S. Raghavendra, 2007. Spray and focus: Efficient mobility-assisted routing for heterogeneous and correlated mobility. Proceedings of the 5th Annual IEEE International Conference on Pervasive Computing and Communications Workshops, Mar. 19-23, IEEE Xplore Press, White Plains, NY., pp: 79-85.

DOI: 10.1109/PERCOMW.2007.108

Suganthe, R. and P. Balasubramanie, 2008. Efficient routing for intermittently connected mobile ad hoc network. Int. J. Comput. Sci. Netw. Security, 8: 184-191.

Supriya, T. and C. Pramila, 2014. Analytic study of spray and wait routing protocol in delay tolerant network. Int. J. Adv. Technol. Eng. Sci.

Vahdat, A. and D. Becker, 2000. Epidemic routing for partially connected ad hoc networks. Technical Report CS-200006, Duke University.

Warthman, F., 2012. Delay and Disrpution Tolerant Networks (DTNs).

Xue, L., J. Liu and J. Peng, 2012. An adaptive message ferry routing algorithm for delay tolerant networks. Proceedings of the IEEE 14th International Conference on Communication Technology, Nov. 9-11, IEEE Xplore Press, Chengdu, pp: 699-703. DOI: 10.1109/ICCT.2012.6511295

Yu, D. and Y.B. Ko, 2009. FFRDV: Fastest-ferry routing in DTN-enabled vehicular ad hoc networks. Proceedings of the 11th International Conference on Advanced Communication Technology, Feb. 15-18, IEEE Xplore Press, Phoenix Park, pp: 1410-1414.

Zhang, Z., 2006. Routing in intermittently connected mobile ad hoc networks and delay tolerant networks: Overview and challenges. IEEE Commun. Surveys Tutorials, 8: 24-37. DOI: 10.1109/COMST.2006.323440

Zhang, Z., M. Ma and Z. Jin, 2014. CCS-DTN: Clustering and network coding-based efficient routing in social DTNs. Sensors, 15: 285-303. DOI: $10.3390 / \mathrm{s} 150100285$

Zhao, W., M. Ammar and E. Zegura, 2004. A message ferrying approach for data delivery in sparse mobile ad hoc networks. Proceedings of the 5th ACM International Symposium on Mobile $\mathrm{Ad} \mathrm{Hoc}$ Networking and Computing, May 24-26, ACM, Tokyo, Japan, pp: 187-198. DOI: $10.1145 / 989459.989483$ 